\title{
ACTIVIDAD FÍSICA Y ALIMENTACIÓN EN ESTUDIANTES UNIVERSITARIOS CHILENOS
}

\author{
PHYSICAL ACTIVITY AND EATING BEHAVIORS AMONG CHILEAN \\ UNIVERSITY STUDENTS
}

\author{
ATIVIDADE FÍSICA E ALIMENTAÇÃO EM ESTUDANTES \\ UNIVERSITARIOS CHILENOS
}

\author{
Aileen G. Chales-Aoun* \\ José M. Merino Escobar**
}

\begin{abstract}
RESUMEN
La sociedad occidental se caracteriza actualmente por comportamientos sedentarios en el trabajo, actividades domésticas, recreativas y de ocio, así también por la ingesta calórica desequilibrada y consumo indiscriminado de sal, azúcar y grasas saturadas. Chile no está ajeno a esta realidad: casi dos tercios de la población tiene sobrepeso, además de no practicar deportes ni actividad física. Objetivo: Describir los comportamientos alimentarios y la actividad física de estudiantes matriculados en programas de pregrado en ciencias de la salud y ciencias humanas en una universidad chilena, ubicada en la zona centro-sur del país. Material y método: Se realizó un estudio transversal, cuantitativo, con una muestra de 331 estudiantes, hombres y mujeres (20-24 años) que cursaban el tercer ańo de carreras de pregrado de una universidad del centro sur de Chile. Esta investigación utilizó la escala validada de origen colombiano "CEVJU-R2". Resultados: La mayoría de los y las estudiantes reconocieron tener comportamientos alimentarios poco saludables y más de la mitad desarrollan bajos niveles de actividad física, independiente de si cursaba una carrera de pregrado relacionada o no con la salud. Sin embargo, los y las estudiantes de profesiones de la salud obtienen levemente mejores puntajes en relación con sus pares de ciencias humanas. Conclusión: Los estudiantes universitarios, así como la población chilena, mantienen prácticas de actividad física y patrones de alimentación poco saludables.
\end{abstract}

Palabras clave: Estilo de vida saludable; Conductas de salud; Actividad física, Hábitos alimenticios; Estudiantes.

\begin{abstract}
Western society is currently characterized by sedentary behavior at work, domestic, recreational and leisure activities, as well as unbalanced caloric intake and indiscriminate consumption of salt, sugar and saturated fats. Chile is no stranger to this reality: almost two thirds of the population is overweight, in addition to not practicing sports or any physical activity. Objective: To describe the eating behaviors and the physical activity
\end{abstract}

*Socióloga. Colaboradora académica Departamento de Sociología. Facultad de Ciencias Sociales. Universidad de Concepción, Chile.Email: aileenchales@udec.cl. Autor de correspondencia.

${ }^{* *}$ Sociólogo. Profesor Departamento de Sociología. Facultad de Ciencias Sociales. Universidad de Concepción, Chile. Email jmerino@udec.cl 
of students enrolled in undergraduate programs in health sciences and human sciences at a Chilean university, located in the central-southern part of the country. Materials and methods: A cross-sectional, quantitative study was conducted with a sample of 331 students, men and women (20-24 years old), who were in the third year of undergraduate programs at a university in central-southern Chile. This research used the validated scale of Colombian origin "CEVJU-R2". Results: Most university students reported having unhealthy eating behaviors and more than half of them show low levels of physical activity, regardless of whether their undergraduate programs were related to health sciences or not. However, students of health-related professions scored slightly better in relation to their peers in the humanities. Conclusion: University students, as well as the Chilean population, show low levels of physical activity and unhealthy eating patterns.

Key words: Healthy lifestyle, Health behaviors, Physical activity, Eating behavior, Students.

\section{RESUMO}

A sociedade ocidental é caracterizada atualmente por comportamentos sedentários no trabalho, atividades domésticas, recreativas e de lazer, assim como pela ingestão calórica desequilibrada e consumo indiscriminado de sal, açúcar e gorduras saturadas. Chile não é estranho a essa realidade: quase dois terços da população está com excesso de peso, além de não praticar esportes ou atividade física. Objetivo: Descrever os comportamentos alimentares e a atividade física de estudantes matriculados em programas de graduação em ciências da saúde e ciências humanas em uma universidade chilena, localizada na parte centro-sul do país. Material e método: Foi realizado um estudo transversal, quantitativo, com uma amostra de 331 estudantes, homens e mulheres $(20$ a 24 anos), que cursavam o terceiro ano de carreira em uma universidade do sul do Chile. Esta pesquisa utilizou a escala validada de origem colombiana "CEVJU-R2". Resultados: A maioria dos e das estudantes reconheceram ter comportamentos alimentares não saudáveis e mais da metade desenvolvem baixos níveis de atividade física, independentemente da área da carreira de graduação, relacionada ou não à saúde. Não obstante, os estudantes de profissôes da saúde obtêm pontuações ligeiramente melhores em relação aos colegas de ciências humanas. Conclusão: Estudantes universitários, assim como a população chilena, mantêm práticas de atividade física e padrôes alimentares pouco saudáveis.

Palavras-chave: Estilo de Vida Saudável; Comportamentos Relacionados com a Saúde; Atividade Física; Comportamento Alimentar; Estudantes.

Fecha recepción: 22/10/2018

Fecha aceptación: 22/06/2019

\section{INTRODUCCIÓN}

Cuando las personas se refieren a la salud, generalmente piensan sólo en los aspectos físicos, raramente en los aspectos psicológicos y conductuales asociados. No obstante, en la actualidad es necesario considerar los aspectos sociales, económicos, culturales y espirituales de los individuos $^{(1)}$. El problema de investigación surge a partir del aumento generalizado y sostenido de los niveles de sedentarismo, sobrepeso y obesidad en los últimos años. Actualmente, al menos un $60 \%$ de la población mundial no realiza la actividad física necesaria para obtener los beneficios que ésta conlleva $^{(2)}$. Ya en 2016, el 39\% de las personas de 18 o más años tenían sobrepeso, y el $13 \%$ eran obesas ${ }^{(3)}$. En 2018, en Chile, el 81,3\% de la población adulta se encuentra inactivo físicamente ${ }^{(4)}$ y el $34,4 \%$ es obeso, ubicando a Chile como el segundo país de la OCDE con la tasa más alta de obesidad ${ }^{(5)}$, el tercer lugar en América Latina de personas con sobrepeso, y el primero del continente en obesidad infantil ${ }^{(6)}$.

Los beneficios de la actividad física han sido respaldados por pruebas científicas que vinculan el aumento de ejercicio y los estilos de vida saludables con una mejor salud y calidad de vida ${ }^{(2,7,8)}$. Por su 
parte, los hábitos alimentarios, además de satisfacer las necesidades físicas, favorecer el funcionamiento diario del organismo, el desarrollo de las funciones vitales y el estado de salud, influyen directamente en la aparición de enfermedades cardiovasculares y no transmisibles, diabetes y cáncer ${ }^{(9-14)}$. De igual manera, la alimentación y la actividad física influyen en la salud, en forma independiente o combinada $^{(7,15)}$, existiendo correlación positiva entre alimentación saludable y participación en actividad física y deporte ${ }^{(7,16-19)}$. Por su parte, las instituciones de educación superior juegan un papel importante en el establecimiento de conocimientos, conductas y actitudes que promuevan el desarrollo de cada individuo desde una perspectiva integradora de sus dimensiones biológica y psicosocial. De esta manera, la detección a tiempo de estos fenómenos, sobre todo en la población joven, podría contribuir de forma directa en la salud, el bienestar y la calidad de vida de las personas.

Tradicionalmente en las ciencias sociales y de la conducta, el comportamiento con efectos en la salud de las personas ha sido específicamente observado, descrito y explicado como un evento discreto, más que como un proceso o una secuencia de etapas ${ }^{(20)}$. A diferencia de otras teorías, el Modelo
Transteórico (MTT) ${ }^{(21)}$ (Figura 1) postula que el cambio de conducta no es un fenómeno binario ${ }^{(22)}$ sino que se trata de una secuencia temporal que se logra a través de la integración de procesos y principios del cambio, presentes en distintas teorías de intervención ${ }^{(23)}$.

Esta investigación tuvo como objetivo describir las conductas de alimentación y actividad física de jóvenes universitarios de tercer año de una universidad del centro-sur de Chile. Lo anterior, a través de la descripción de las prácticas de actividad física y alimentación, las motivaciones asociadas, el nivel de satisfacción relacionado y los recursos disponibles para implementarlas. Además, se intentó configurar estas conductas de salud según el área de inserción disciplinaria del estudiante, comparando carreras del área de la salud respecto de carreras de las ciencias humanas.

Se considera como hipótesis de investigación, por tanto, que i) En general, los estudiantes universitarios de tercer año desarrollan prácticas de alimentación y actividad física no saludables, y ii) los estudiantes universitarios de carreras de salud tienen mejores prácticas de alimentación y actividad física que aquellos que estudian carreras de las ciencias humanas.

Figura 1. Adaptación de "Representación de las etapas de cambio del MTT"

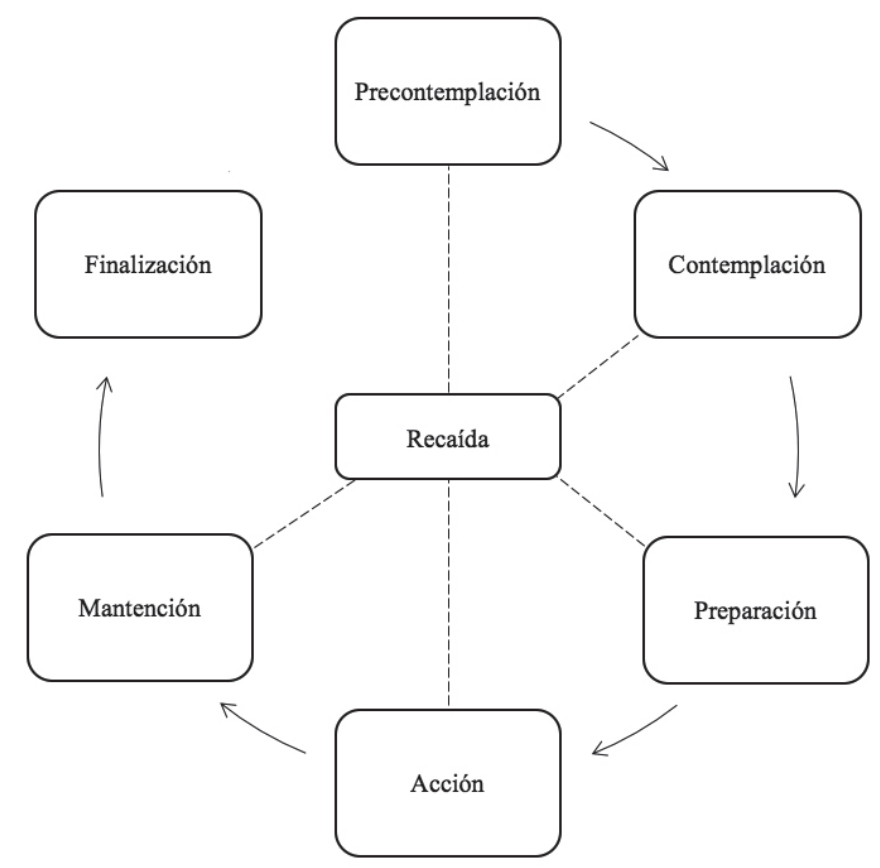

Fuente: Adaptado de Cabrera ${ }^{(21)}$. 


\section{MATERIAL Y MÉTODO}

Se realizó un estudio cuantitativo, descriptivo y transversal. Se realizó un muestreo no probabilístico. La Universidad del centro-sur chileno dispone de 7 carreras de pregrado ligadas a la salud, en particular de conductas de actividad física y alimentación, a saber: Enfermería, Obstetricia y Puericultura, Odontología, Pedagogía en Educación Física, Kinesiología, Medicina, y Nutrición y Dietética. De estas, solo las últimas cuatro mencionadas accedieron a participar de la investigación. La aplicación de los cuestionarios se realizó previa coordinación con las respectivas jefaturas de carrera, tanto de salud como de ciencias humanas. Los criterios de inclusión corresponden a edad de 20 a 24 años y año de ingreso (2015) de las y los estudiantes. Fue así como la muestra del estudio estuvo conformada por 331 estudiantes, hombres y mujeres de 20 a 24 años de edad, que cursaran tercer año de carreras de salud y ciencias humanas (Tabla 1) quienes participaron de manera voluntaria, firmando un formulario de Consentimiento Informado que fue revisado y aprobado por el Comité de Ética de la Facultad de Medicina de la Universidad de Concepción. Los cuestionarios fueron aplicados a los y las estudiantes durante el segundo semestre de 2017, siendo administrados y analizados exclusivamente por la investigadora, utilizados únicamente para fines académicos.
A nivel internacional, son varios los instrumentos que permiten evaluar las conductas promotoras de la salud en la población. No obstante, ninguno ha sido adaptado para población latina y menos a población universitaria. En consecuencia, un equipo de investigación de Salud y Calidad de Vida de la Pontificia Universidad Javeriana de Cali, Colombia (24), creó el "Cuestionario de Estilos de Vida en Jóvenes Universitarios” (CEVJU), que logró reunir las propiedades psicométricas adecuadas para convertirse en una alternativa interesante y plausible en la evaluación del estilo de vida, la detección de necesidades en el ámbito de la salud y el desarrollo de estrategias para su intervención desde las instituciones educativas, particularmente de las conductas de salud en jóvenes pertenecientes a educación superior ${ }^{(25)}$. Para la presente investigación, y contando con la autorización por parte de sus autores, se utilizó la versión original del CEVJU-R2. Este instrumento ${ }^{(26)}$, además de contar con un apartado de datos sociodemográficos, contempla siete dimensiones de los estilos de vida: actividad física, tiempo de ocio, alimentación, consumo de alcohol, cigarrillo y drogas ilegales; sueño, afrontamiento y sexualidad. Se consideran cuatro aspectos relacionados con cada dimensión del estilo de vida: las prácticas, las motivaciones (para realizar y para no realizar dichas prácticas), los recursos (personales y externos) que posibilitan o dificultan dichas prácticas, y la etapa de cambio en

Tabla 1. Distribución de la muestra según grupo de carreras.

\begin{tabular}{lrr}
\hline & n & \% \\
\hline Carreras del área de la salud (G1) & 176 & 53,2 \\
Pedagogía en Educación Física & 58 & 17,5 \\
Nutrición y Dietética & 30 & 9,1 \\
Medicina & 67 & 20,2 \\
Kinesiología & 21 & 6,3 \\
\hline Carreras de las ciencias humanas (G2) & 155 & 46,8 \\
Educación General Básica & 21 & 6,3 \\
Administración Pública y Ciencia Política & 17 & 5,1 \\
Ingeniería Comercial & 38 & 11,5 \\
Trabajo Social & 47 & 14,2 \\
Arquitectura & 32 & 9,7 \\
\hline Total & 331 & 100 \\
\hline
\end{tabular}


la que se encuentra (si considera o no hacer cambios en la conducta). Para este estudio se recolectaron datos sociodemográficos, de salud, académicos y familiares, además de solo dos dimensiones del CEVJU-R2: actividad física y alimentación.

La definición conceptual de actividad física es considerada como cualquier movimiento corporal producido por los sistemas musculares y esqueléticos que exija gasto de energía, mientras que la de alimentación corresponde al conjunto de acciones mediante las cuales se proporcionan alimentos al organismo. Un total de 3 ítems de prácticas de actividad física (3 a 12 puntos en total; punto medio en 7,5 puntos), y 7 ítems de prácticas de alimentación (7 a 28 puntos en total; punto medio en 17,5), considerando puntuaciones posibles de 1 (siempre) a 4 (nunca) para cada ítem. Se categorizó como "práctica no saludable" (mayor a punto medio) y "práctica saludable" (menor a punto medio) según el puntaje total obtenido en cada dimensión.

Los datos contenidos en cada uno de los cuestionarios fueron ingresados y posteriormente procesados con el software IBM SPSS Statistics (Statistical Package for the Social Sciences), versión 21 para OS X. Los antecedentes sociodemográficos, de salud, académicos y familiares de los estudiantes, además de las motivaciones, recursos y etapas del cambio (ítems AF7 y AL11) fueron analizados por medio de las frecuencias obtenidas. Se realizó un análisis descriptivo para las preguntas sobre prácticas de alimentación y actividad física, para posteriormente ser analizados comparando los grupos de estudiantes del área de ciencias de la salud (Grupo 1) versus los de las ciencias humanas (Grupo 2), según la escala estipulada por el CEVJU-R2, considerando la puntuación obtenida total y el punto medio de la variable.

Además, se realizaron pruebas $\mathrm{T}$ de Student para muestras independientes, para comparar las medias del Grupo 1 y Grupo 2. Para analizar las variables categóricas según las variables dependientes "Prácticas de Actividad física" (AF) y "Prácticas de Alimentación" (AL), se realizaron pruebas de Chi-Cuadrado de Pearson con nivel de error del 5\%. Se realizó la misma operación para la variable independiente "Área de la carrera" (G1 y G2).

\section{RESULTADOS}

En el estudio participaron 331 estudiantes de tercer año de carreras de pregrado. Considerando que la totalidad de la muestra cursaba el tercer año de la carrera (año de ingreso 2015), la mayoría tenía 20 o 21 ańos de edad al momento de participar en la investigación. Fueron omitidos los cuestionarios que tenían otro año de ingreso, o bien aquellos estudiantes que tuvieran 25 años o más, considerando las edades estipuladas en la definición de juventud de la Organización Mundial de la Salud ${ }^{(27)}$. En este contexto, sólo el 3,6\% declaró ser padre o madre de un hijo/a; el 99\% de los casos reconoció ser soltero/a como su estado civil; no obstante, el 42,6\% declaró tener pareja. Poco más de la mitad de la muestra $(55,5 \%)$ eran mujeres. La gran mayoría tenía una procedencia geográfica urbana $(84,6 \%)$ y sólo el $14,8 \%$ trabajaba y estudiaba al momento de responder el cuestionario.

Al solicitarles que autoevaluaran su estado de salud actual, la gran mayoría calificó de 5 a 7 (74\%), pero un preocupante 26\% lo hizo de manera baja a regular (1 a 4). El 80,6\% afirmó no tener ninguna enfermedad diagnosticada, mientras que, del porcentaje restante, dos tercios tenía alguna enfermedad crónica de las vías respiratorias o bien alguna enfermedad crónica a nivel endocrino, nutricional, metabólico o del sistema digestivo. En general, tras realizar el cálculo con los pesos y estaturas informados por los estudiantes, el 67,7\% se ubicó en la categoría de peso normal. Si bien esta cifra incluye una gran parte de la muestra, es igualmente notorio el hecho que casi el 30\% de estos jóvenes universitarios se encuentre con sobrepeso u obesidad.

Según las frecuencias obtenidas sobre las prácticas de actividad física, es importante destacar el porcentaje que aseguró caminar o andar en bicicleta en lugar de utilizar otro medio de transporte, al desplazarse a un lugar cercano de manera frecuente $(75,3 \%)$, o bien hacer algún ejercicio o práctica corporal durante al menos 30 minutos tres veces a la semana $(52,9 \%)$. No obstante, de acuerdo a la puntuación y categorización asignada a las conductas de actividad física y alimentación evaluadas por el CEVJU-R2, el 51,8\% de los estudiantes tiene "prácticas de actividad física no 
saludables" (Tabla 2). Esto se explica por la gran cantidad de jóvenes universitarios que no hace ejercicio físico (o realiza pocas veces) durante 30 minutos tres veces a la semana $(47,2 \%)$, además del alto porcentaje $(74,2 \%)$ que aseguró no practicar ningún deporte.

Por su parte, la clasificación de las conductas de alimentación de los jóvenes universitarios es aún más alarmante: casi el $97 \%$ de los participantes se ubicaron en prácticas no saludables. Al evaluar cada ítem por separado, si bien cerca del $98 \%$ de los casos aseguró no vomitar o tomar laxantes después de comer en exceso, las demás prácticas evaluadas arrojaron resultados consistentes considerando una semana habitual del estudiante.

Utilizando como criterio de clasificación la división por grupos de carrera, Grupo 1 (Carreras de la salud) y Grupo 2 (Carreras de las ciencias humanas), los promedios de los puntajes obtenidos en las preguntas sobre prácticas de actividad física fueron significativamente mayores (peores, según la escala) en el Grupo 2, con una diferencia de 8,28 vs 6,85 . Respecto de los promedios de los puntajes obtenidos en las preguntas sobre prácticas de alimentación, el Grupo 1 obtuvo una leve pero significativa diferencia respecto del grupo de estudiantes del área humanista $(21,8$ vs 21,38$)$ (Tabla 3).
El principal motivo, tanto para realizar actividad física como para alimentarse adecuadamente, fue "beneficiar" o "mantener" la salud, con un 52,9 y $50,8 \%$ de frecuencias, respectivamente. Por el contrario, la razón más reconocida por los jóvenes universitarios para no realizar actividad física es la "pereza", con un 63,8\%. En el caso de la alimentación adecuada, el principal motivo para no hacerlo fue "no te gusta restringirte en lo que comes" (40,3\%).

Los recursos con los que cuenta una persona, materiales o inmateriales, son fundamentales para cada una de las acciones que realiza. En general, más de la mitad de los estudiantes consideró tener buen estado de salud para realizar actividad física $(55,9 \%)$. Respecto al recurso "conocimiento" para realizar prácticas saludables, dos tercios de los participantes reconoció tener conocimiento para elegir alimentos saludables, mientras que sólo un tercio aseguró tener conocimiento para realizar adecuadamente actividad física o ejercicio. Según el criterio de división de los grupos, es decir, el área de la carrera, los estudiantes del área de la salud que afirmaron contar con este recurso, para ambas prácticas, duplicaron a quienes provenían de otras carreras (70 vs 30\%). A la vez, según los jóvenes universitarios, este recurso parece ser crucial para las prácticas saludables de actividad física y alimentación.

Tabla 2. Frecuencia y porcentaje de clasificación de prácticas según puntaje obtenido en cada dimensión.

\begin{tabular}{lrr}
\hline & n & \% \\
\hline Prácticas de Actividad Física $(\mathrm{n}=328)$ & & \\
No saludable & 170 & 51,8 \\
Saludable & 158 & 48,2 \\
\hline Prácticas de Alimentación $(\mathrm{n}=317)$ & & \\
No saludable & 307 & 96,8 \\
Saludable & 10 & 3,2 \\
\hline
\end{tabular}

Tabla 3. Diferencia de medias de puntajes obtenidos en ítems de prácticas de AF y AL, según grupo de carrera.

\begin{tabular}{|c|c|c|c|c|c|c|}
\hline & \multicolumn{2}{|c|}{ Grupo 1} & \multicolumn{2}{|c|}{ Grupo 2} & \multirow[b]{2}{*}{ Prueba t } & \multirow[b]{2}{*}{ Sig. } \\
\hline & $\mathrm{M}_{\mathrm{G} 1}$ & $\mathrm{SD}_{\mathrm{G} 1}$ & $\mathrm{M}_{\mathrm{G} 2}$ & $\mathrm{SD}_{\mathrm{G} 2}$ & & \\
\hline Puntajes Prácticas AF & 6,85 & 2,42 & 8,28 & 1,98 & $-5,908$ & 0,000 \\
\hline Puntajes Prácticas AL & 21,8 & 1,69 & 21,38 & 2,04 & 2,001 & 0,046 \\
\hline
\end{tabular}

Prueba t para muestras independientes. 
Considerando el Modelo Transteórico, la mayoría de los jóvenes universitarios se reconocieron en la etapa de contemplación respecto a sus prácticas de actividad física $(40,2 \%)$ y las de alimentación (36,7\%) (Tabla 4), es decir, "aquellos que podrían mejorar, pero no saben si lo harán", pero que, según el Modelo, podrían tomar acción dentro de 6 meses.

Tabla 4. Etapas de cambio en que se ubicaron los estudiantes según dimensión (\%).

\begin{tabular}{lccccc}
\hline & Precontemplación & Contemplación & Preparación & Acción & Mantención \\
\hline Actividad Física & $13,7 \%$ & $40,2 \%$ & $17,4 \%$ & $23,8 \%$ & $4,9 \%$ \\
Alimentación & $11,6 \%$ & $36,7 \%$ & $17,1 \%$ & $30,0 \%$ & $4,6 \%$ \\
\hline
\end{tabular}

\section{DISCUSIÓN}

Al igual que el fenómeno a nivel mundial ${ }^{(2,3)}$ y nacional ${ }^{(4,5)}$ los estudiantes universitarios que participaron en la investigación presentaron prácticas no saludables de actividad física y alimentación, detectándose además diferencias significativas entre los grupos comparados. No obstante, la mayoría declara tener buen estado de salud (en los ítems de antecedentes o como recurso para realizar dichas prácticas). Lo anterior, es consistente con la realidad del país, donde sólo el 31,8\% califica como practicantes de actividad física y deportiva. Para el rango de edad de 18 a 29 años, el 50,4\% no realiza actividad física ${ }^{(4)}$. Por su parte, el $86,7 \%$ es sedentario, es decir, personas que en los últimos 30 días no practicaron deporte o realizaron actividad física fuera de su horario de trabajo, durante $30 \mathrm{o}$ más minutos cada vez, 3 o más veces por semana (73,5 y 81,2\% para los rangos de edad de 15 a 19 años, y de 20 a 29 años, respectivamente). Además, sólo el $15 \%$ del total país consume 5 o más frutas o verduras durante el día ${ }^{(28)}$.

Pese a que una gran cantidad de jóvenes tiene prácticas no saludables en alguna de las dos dimensiones o en ambas, al igual que en otras investigaciones ${ }^{(18,26)}$, la mayoría indicó que la principal motivación para realizar estas acciones de manera óptima era beneficiar o mantener la salud, lo que implica cierto grado de conciencia acerca de los beneficios que conllevan estas prácticas para la salud y la calidad de vida en general. Sería interesante en futuras investigaciones realizar mediciones considerando la dimensión de Estado
Emocional Percibido, incluido en el instrumento original, para medir esta conducta de salud por separado, y relacionarla a la vez con las prácticas de actividad física y/o alimentación. Otra dimensión interesante a considerar sería el uso o manejo del tiempo libre, puesto que los motivos principales para no realizar las prácticas saludables fueron la pereza y, por otro lado, la falta de tiempo.

Al igual que otros estudios ${ }^{(20)}$, los resultados encontrados en esta investigación revelan la necesidad de que las universidades tomen un rol protagonista en la adquisición de hábitos alimenticios adecuados en sus estudiantes, además de entregar una amplia oferta de espacios que permita la práctica de actividad física regular en el tiempo libre, logrando disminuir los factores de riesgo en salud que se presentan en la etapa universitaria y evitar su empeoramiento en la vida adulta, una vez finalizado este periodo.

Dado que los estudiantes pasan gran parte de su tiempo en la universidad, ésta se convierte en un espacio privilegiado en el que se pueden promover hábitos, entornos físicos y sociales que promuevan la salud y la calidad de vida de sus estudiantes ${ }^{(29)}$. Los resultados de este estudio pueden animar y orientar una reflexión sobre la mejora en los estilos de vida de los estudiantes y su desarrollo integral y, de esta forma, contribuir al mejoramiento de la escuela en términos de impacto positivo, mediados por los procesos curriculares, de planeación, de gestión educativa, y demás componentes del proceso formativo. Es importante generar ambientes de aprendizaje conducentes a la adquisición de hábitos saludables en los estudiantes, fortalecer 
la promoción de la salud, y crear espacios para la apropiación de principios de calidad y estilos de vida saludable ${ }^{(26)}$.

Frente a la posible intervención asociada a los estudiantes y sus prácticas de actividad física y alimentación, la responsabilidad individual sólo puede tener su efecto completo cuando las personas tienen acceso a un estilo de vida saludable, y son apoyados para tomar decisiones saludables ${ }^{(3)}$. No obstante, según los mismos autores del modelo, el paciente es quien realiza el $99 \%$ del trabajo aún no estando en la sala de consulta. Si bien es cierto que muchas personas necesitan ayuda para cambiar, la clave siempre es utilizar la estrategia correcta en el momento adecuado ${ }^{(30)}$.

Tanto la salud como el bienestar físico y la calidad de vida de las personas se ven fuertemente influenciadas por las conductas de salud, y de esta manera, por el estilo de vida de las personas. En otras palabras, cada una de las prácticas de las personas ejerce cierto grado de control sobre el estado de su salud, por lo que, para mantenerse saludable, las personas deben hacer ejercicio, reducir la ingesta de grasa, abstenerse de fumar, mantener la presión arterial baja ${ }^{(2,3,7,8)}$ y desarrollar formas eficaces de manejar los factores estresantes. Mediante el manejo de sus conductas de salud, las personas pueden vivir más tiempo, ser más saludables, y retardar el proceso de envejecimiento. En síntesis, la autogestión es buena medicina, por lo que si los enormes beneficios de estas pocas conductas estuvieran contenidos en una píldora sería declarada un hito científico en el campo de la medicina ${ }^{(31)}$. Sin embargo, he aquí la trampa de la promoción de la salud pues, si no se experimenta el beneficio deseado justo después de realizar un nuevo comportamiento de salud, se reduce la probabilidad de que el nuevo comportamiento se convierta en un hábito. El gran y perdurable desafío en el cambio de hábitos de salud es garantizar que las consecuencias deseadas se experimenten de inmediato y con frecuencia, cuando y donde se lleve a cabo la acción deseada. Entonces, ¿cómo las personas cambian sus conductas? Por un lado, cada persona puede o no saber lo que debería hacer, pero hacer un esfuerzo sostenido para cambiar es un asunto completamente diferente; es trabajo de los investigadores en ciencias sociales en general, apoyados por las teorías y modelos psicológicos, el buscar comprender las conductas de salud visualizándolas como sistemas organizados, compuestos por secuencias de acciones altamente rutinizadas, consecuencias y reacciones que conducen a un resultado predecible ${ }^{(32)}$.

Respecto al Modelo Transteórico, es posible presentarlo como un paradigma de acción que ha dominado los programas de cambio de comportamiento durante las últimas tres o cuatro décadas, siendo utilizados distintos comportamientos en salud (sedentarismo, dietas ricas en grasas, sobrexposición al sol, consumo de tabaco, etc.) ${ }^{(30)}$. Sin duda, el MT es valioso porque se enfoca en el individuo y evalúa su disposición para cambiar, reconociendo el progreso de las personas a través de diferentes etapas de cambio y ayudando a este a reconocerse en alguna de ellas, para luego poder desarrollar intervenciones apropiadas y efectivas con el fin de aumentar la motivación del individuo para cambiar y progresar a la siguiente etapa ${ }^{(33)}$.

\section{CONCLUSIÓN}

En general, al igual que la realidad de la población adulta a nivel mundial y nacional, los estudiantes universitarios que participaron en la investigación presentaron prácticas no saludables de actividad física y alimentación, reportando además diferencias significativas en las variables prácticas de actividad física y alimentación bajo el criterio de división entre el grupo de carreras del área de la salud y las carreras de las ciencias humanas.

Como recomendaciones a considerar para próximas investigaciones, se reconocen varias opciones a nivel de metodología, diseño y muestreo. En primer lugar, sería interesante complementar las prácticas estudiadas por el CEVJU-R2 con las demás dimensiones de la escala, las cuales fueron omitidas en esta oportunidad. Lo anterior, sin descartar la posibilidad de seguir el camino del multimétodo para la recopilación de los antecedentes y conductas declarados por los estudiantes, de manera de complementar la información arrojada por la escala utilizada, y profundizar acerca de las prácticas, motivos, recursos y niveles de satisfacción que tienen sobre sus conductas de actividad física y alimentación. En cuanto al diseño de investigación, una vía atractiva es la de pasar de un estudio transversal a longitudinal, estudiando distintos puntos a lo largo de la carrera universitaria. Por otro lado, incrementar el nivel de análisis, 
transformándola a un modelo multivariado. Esto, conectándose con la posibilidad de expansión de la muestra, ya sea sumando otros cursos de las carreras

\section{REFERENCIAS}

1. Oblitas L. Enciclopedia de psicología de la salud. Bogotá: PSICOM; 2007.

2. Organización Mundial de la Salud [Internet]. Ginebra: Organización Mundial de la Salud; 2018. Actividad Física; 2018 Feb [citado 2018 Oct 3]; [alrededor 1 pantalla]. Disponible en: https://www. who.int/es/news-room/fact-sheets/detail/physicalactivity

3. World Health Organization [Internet]. Geneva: World Health Organization; 2018. Obesity and overweight; 2018 Oct [cited 2018 Oct 5]; [about 1 screen]. Available from: https://www.who.int/ en/news-room/fact-sheets/detail/obesity-andoverweight

4. Ministerio del Deporte, Chile. Resumen ejecutivo Encuesta Nacional de Actividad Física y Deporte 2018 en Población de 18 años y más [Internet]. Santiago: Ministerio del Deporte; 2018 Dic [citado 20 Ene 2019]. Disponible en: http://www.mindep. cl/encuesta-actividad-fisica-y-deporte-2018

5. FAO, FIDA, UNICEF, PMA, OMS. El Estado de la Seguridad Alimentaria y la Nutrición en el Mundo. Fomentando la resiliencia climática en aras de la seguridad alimentaria y la nutrición. [Internet]. Roma: Organización de las Naciones Unidas para la Alimentación y la Agricultura; 2018 [citado 5 Jul 2018]. 218 p. Disponible en: http:// www.fao.org/3/I9553ES/i9553es.pdf

6. FAO, OPS. Panorama de la Seguridad Alimentaria y Nutricional en América Latina y el Caribe [Internet]. Santiago: Organización Panamericana de Salud; 2017 [citado 25 Ene 2018]. 174 p. Disponible en: http://www.fao.org/3/a-i6747s.pdf

7. Organización Mundial de la Salud. Estrategia mundial sobre régimen alimentario, actividad física y salud [Internet]. Ginebra: Organización Mundial de la Salud; 2004 [citado 20 Oct 2017]. 24 p. Disponible en: https://www.who.int/ dietphysicalactivity/strategy/eb11344/strategy_ spanish_web.pdf

8. OMS. Documentos Básicos [Internet]. Ginebra: Organización Mundial de la Salud; 2014 Dic [citado 17 Jul 2017]. 224 p. Disponible en: http:// apps.who.int/gb/bd/PDF/bd48/basic-documents48th-edition-sp.pdf\#page= 1

9. Oblitas L. Psicología de la salud: Una ciencia estudiadas, o incluso como comparación entre universitarios de distintas casas de estudio a nivel local o internacional.

del bienestar y la felicidad. Av. Psicol. [Internet]. 2008 Ene-Dic [citado 9 Jul 2017]; 16(1): 9-38. Disponible en: http://www.unife.edu.pe/pub/ revpsicologia/psicologiasalud.pdf

10. Lema L, Salazar I, Varela M, Tamayo J, Rubio A, Botero A. Comportamiento y salud de los jóvenes universitarios: satisfacción con el estilo de vida. Pensamiento Psicológico. [Internet]. 2009 Ene-Jun [citado 15 Jul 2017]; 5(12): 71-87. Disponible en: http://www.redalyc.org/articulo. oa?id $=80111899006$

11. Mönckeberg F, Muzzo S. La desconcertante epidemia de obesidad. Rev Chil Nutr [Internet]. 2015 Mar [citado 6 Jun 2017]; 42(1): 96-102. Disponible en: https://scielo.conicyt.cl/pdf/rchnut/ v42n1/art13.pdf

12. WHO [Internet]. Geneva: World Health Organization; c2019. Healthy diet; 2018 Oct [cited $2017 \mathrm{Jul}$ 6]; [about 1 screen]. Available from: https://www.who.int/en/news-room/fact-sheets/ detail/healthy-diet

13. WHO. Noncommunicable Diseases Progress Monitor [Internet]. Geneva: World Health Organization; 2017 [cited 2017 Jul 21]. 234 p. Available from: http://apps.who.int/iris/bits tream/10665/258940/1/9789241513029-eng. pdf?ua $=1$

14. WHO [Internet]. Geneva: World Health Organization; c2019. 10 facts on obesity; 2017 Oct [cited 2017 Jul 23]; [about 1 screen]. Available from: https:/www.who.int/features/factfiles/obesity/en/

15. Marádová E. A new concept of education to healthy eating habits in primary school. School and Health. [Internet]. 2011 [cited 2017 Jul 20]; 21: 155-165. Available from: http://www.ped.muni. cz/z21/knihy/2011/40/texty/eng/13_maradova_ eng.pdf

16. Balaguer I. Estilos de vida en la adolescencia. Valencia: Promolibro. 2002. 260 p.

17. WHO. The World Health Report 2002. Reducing Risks, Promoting Healthy Life [Internet]; 2002 [cited 2017 Jul 10]. 230 p. Available from: https:// www.who.int/whr/2002/en/whr02_en.pdf

18. Castillo I, Balaguer I, García-Merita M. Efecto de la práctica de actividad física y de la participación deportiva sobre el estilo de vida saludable en la adolescencia en función del género. Revista de Psicología del Deporte. [Internet]. 2007 [citado 12 
Jul 2017]; 16(2): 201-210. Disponible en: https:// www.redalyc.org/articulo.oa?id=235119266001

19. Espinoza L, Rodríguez F, Gálvez J, MacMillan N. Hábitos de alimentación y actividad física en estudiantes universitarios. Rev Chil Nutr. [Internet]. 2011 Dic [citado 14 Jul 2017]; 38(4): 458-465. Disponible en: https://scielo.conicyt.cl/ pdf/rchnut/v38n4/art09.pdf

20. Prochaska J, Velicer W. The Transtheoretical Model of Health Behavior Change. Am J Health Promot. 1997; 12(1): 38-48.

21. Cabrera G. El modelo transteórico del comportamiento en salud. Revista Facultad Nacional de Salud Pública, 2000; 18(2): 129-138.

22. Prochaska J, DiClemente C. Stages and Processes of Self-Change of Smoking: Toward An Integrative Model of Change. J Consultar Clin Psychol. 1983; 51(3): 390-395.

23. Glanz K, Rimer B, Viswanath K. Health Behavior: Theory, Research, and Practice. 5th ed. San Francisco, CA: Jossey-Bass. 2015. 512 p.

24. Salazar I, Varela M, Tovar J, Cáceres D. Construcción y validación de un cuestionario de factores de riesgo y de protección para el consumo de drogas en jóvenes universitarios. Acta Colombiana de Psicología [Internet]. 2006 [citado 19 Jul 2017]; 9(2): 19-30. Disponible en: https:// www.redalyc.org/articulo.oa?id=79890203

25. Salazar-Torres I, Varela-Arévalo M, Lema-Soto L, Tamayo-Cardona J, Duarte-Alarcón C, Equipo de investigación CEVJU Colombia. Evaluación de las conductas de salud en jóvenes universitarios. Rev. Salud Pública. [Internet]. 2010 [citado 22 Jul 2017]; 12 (4): 599-611. Disponible en: https:// revistas.unal.edu.co/index.php/revsaludpublica/ article/view/33281
26. Campo Y, Pombo L, Teherán A. Estilos de vida saludable y conductas de riesgo en estudiantes de medicina. Rev Salud UIS. [Internet]. 2016 Sep [citado 2017 Jun 24]; 48(3): 301-309. Disponible en: https://revistas.uis.edu.co/index.php/revistasaluduis/ article/view/5667/5832

27. WHO. Young people $\mathbb{S}_{\mathrm{s}}$ health - a challenge for Society. [Internet]. Geneva: World Health Organization; 1986 [cited 2017 Ago 3]. 120 p. Available from: http://apps.who.int/iris/ bitstream/10665/41720/1/WHO_TRS_731.pdf

28. Ministerio de Salud, Chile. Informe Encuestas. Encuesta Nacional de Salud 2016-2017. Primeros Resultados [Internet]. Santiago: Departamento de Epidemiología Ministerio de Salud Gobierno de Chile; c2019 [citado 13 Ene 2018]. Disponible en: http://epi.minsal.cl/wp-content/ uploads/2018/03/1\%C2\%BA-Resultados-ENS_ DEPTO.EPIDEMIOLOGIA.MINSAL.pdf

29. Varela-Arévalo M, Ochoa-Muñoz A, Tovar-Cuevas J. Tipologías de estilos de vida en jóvenes universitarios. Rev Univ. Salud. [Internet]. 2016 [citado 29 Jun 2017]; 18(2):246-256. Disponible en: http://www. scielo.org.co/pdf/reus/v18n2/v18n2a06.pdf

30. Prochaska J, Norcross J, DiClemente C. Changing for Good: A Revolutionary Six-Stage Program for Overcoming Bad Habits and Moving Your Life Positively Forward. Reprint Edition 1994. New York: Harper Collins. 2007. 304 p.

31. Bandura, A. The Primacy of Self-Regulation in Health Promotion. Applied Psychology: An International Review. 2005; 54(2), 245-254.

32. Anderson, N. Encyclopedia of Health and Behavior. California: Sage Publications, Inc.; 2004.

33. Seals JG. Integrating the transtheoretical model into the management of overweight and obese adults. J Am Acad Nurse Pract. 2007; 19 (2): 63-71. 